\title{
Isospin splitting in Wilson chiral perturbation theory for lattice QCD with three non-degenerate quark flavours
}

\section{Sebastian Engelnkemper, Gernot Münster*}

Universität Münster, Institut für Theoretische Physik

Wilhelm-Klemm-Str. 9, D-48149 Münster, Germany

E-mail: engelnkemper@uni-muenster.de, munsteg@uni-muenster.de

We have calculated the expansions of the masses of the pseudoscalar mesons in chiral perturbation theory at next-to-leading order for lattice QCD with three light quark flavours, taking the mass difference between the up and down quarks into account.

The 33rd International Symposium on Lattice Field Theory

14 -18 July 2015

Kobe International Conference Center, Kobe, Japan

*Speaker. 
In Quantum Chromodynamics the masses of quarks are free parameters. The knowledge of their numerical values is of great importance for phenomenological purposes [1]. Particularly delicate is the determination of the small masses of the up and down quarks. Their mass difference contributes significantly to the isospin splitting of hadron masses.

Lattice QCD offers the possibility to study quark masses by systematically investigating the dependence of hadronic observables on the quark masses, see e.g. [2]. An indispensable tool for such kind of studies is chiral perturbation theory, which describes analytically how hadron masses and other quantities depend on the quark masses. The systematic errors entailed by lattice discretisation can be taken into account in chiral perturbation theory, leading to the lattice spacing $a$ being an additional expansion parameter. In the case of Wilson fermions this is called Wilson chiral perturbation theory. For reviews see [3, 4].

The isospin splitting produced by the small mass difference between up and down quarks has been investigated in lattice QCD in recent years $[5,6,7]$. We have calculated the isospin splittings of pseudoscalar meson masses in the framework of Wilson chiral perturbation theory with $N_{\mathrm{f}}=1+1+1$ quark flavours. The purpose of these calculations is to reveal the effects of the lattice discretisation on the isospin splitting for pion and kaon meson masses. In addition to that, even the further limit of vanishing lattice spacing $a$ is of interest, as it includes the effects of mixing in next-to-leading order, which are not completely included in the original work [8].

We have actually performed the calculations for twisted mass lattice QCD, which implements a chirally rotated mass term $[9,10]$. Setting the twist angle to a value of $\pi / 2$ ("full twist") implies automatic $\mathscr{O}(a)$ Symanzik-improvement $[11,12]$. In practice, simulations of twisted mass lattice QCD including strange quarks, are done differently, namely by adding a fourth heavy charm quark and implementing full twist in both the up-down and the strange-charm sector in order to ensure automatic $\mathscr{O}(a)$ improvement in all observables $[13,14,15]$. The introduction of the fourth quark flavour is necessary for this purpose. Our calculations for three flavours explicitely show how the pion masses are influenced by the presence of strange quarks that are not accompanied by charm quarks, and in this way corroborate the inclusion of a fourth quark flavour in the simulations.

The calculations follow the techniques developed in [16, 17, 18, 19, 20, 21]. The masses of the members of the pseudoscalar meson octet have been obtained to next-to-leading order (NLO). The dependence on the lattice spacing $a$ shows up in additional terms proportional to powers of $a$. The small isospin mass splitting $\Delta m=m_{\mathrm{d}}-m_{\mathrm{u}}$ is included to order $\Delta m^{2}$, and lattice effects are included to order $a^{2}$. For details of the set-up and the method see [22].

In NLO the inverse propagator of pseudoscalar mesons is of the form

$$
\mathrm{i} G^{-1}\left(p^{2}\right)=p^{2} \mathbf{1}+\mathfrak{M}_{\mathrm{LO}}-A+p^{2} B,
$$

where $\mathfrak{M}_{\mathrm{LO}}$ is the LO mass squared matrix. The NLO matrices $A$ and $B$ are rather complicated and contain various off-diagonal terms that generate mixings in the $\pi^{0}-\eta$ sector and mixings among the kaon fields. Implementing field renormalisation via

$$
\mathrm{i} G^{-1}\left(p^{2}\right)=\left(\mathbf{1}+\frac{B}{2}\right)\left[p^{2} \mathbf{1}+\mathfrak{M}_{\mathrm{LO}}-A-\frac{1}{2}\left\{B, \mathfrak{M}_{\mathrm{LO}}\right\}\right]\left(\mathbf{1}+\frac{B}{2}\right)+\mathscr{O}\left(p^{6}\right),
$$

the squared meson masses are obtained by diagonalisation of the matrix $\mathfrak{M}_{\mathrm{LO}}-A-\frac{1}{2}\left\{B, \mathfrak{M}_{\mathrm{LO}}\right\}$. With our conventions for the generators of the quark mass splitting the neutral pion is $\pi_{1} \equiv \pi^{0}$, and 
the charged pions $\pi^{ \pm}$are linear combinations of the mass eigenstates $\pi_{2}$ and $\pi_{3}$. For non-vanishing twist angle $\omega$ the charged pions have different masses, which is a pure lattice artifact that vanishes in the continuum limit $a \rightarrow 0$.

The result for the mass splitting between the neutral pion and the charged pion $\pi_{2}$, presented to order $a$ here, is given by

$$
\begin{aligned}
& M_{\pi_{1}}^{2}-M_{\pi_{2}}^{2}=B_{0} \Delta m^{2}\left\{\frac{1}{4\left(m_{\mathrm{s}}-\hat{m}\right)}\left[1-\frac{a W_{0}(1-\cos \omega)}{B_{0}\left(m_{\mathrm{s}}-\hat{m}\right)}\right]\right. \\
& +\frac{4 B_{0}}{3 F_{0}^{2}}\left(-3 L_{4}^{\mathrm{r}}+2 L_{5}^{\mathrm{r}}+6 L_{6}^{\mathrm{r}}-78 L_{7}^{\mathrm{r}}-18 L_{8}^{\mathrm{r}}\right)+\frac{4 B_{0}}{F_{0}^{2}}\left(\frac{\hat{m}}{m_{\mathrm{s}}-\hat{m}}\right)\left(-3 L_{4}^{\mathrm{r}}-2 L_{5}^{\mathrm{r}}+6 L_{6}^{\mathrm{r}}\right) \\
& +\frac{4 a W_{0}}{F_{0}^{2}\left(m_{\mathrm{s}}-\hat{m}\right)}\left[\left(-L_{5}^{\mathrm{r}}-3 W_{4}^{\mathrm{r}}-W_{5}^{\mathrm{r}}+3 W_{6}^{\mathrm{r}}\right)\right. \\
& \quad+\left(L_{4}^{\mathrm{r}}+L_{5}^{\mathrm{r}}+20 L_{7}^{\mathrm{r}}+4 L_{8}^{\mathrm{r}}+2 W_{4}^{\mathrm{r}}+W_{5}^{\mathrm{r}}-3 W_{6}^{\mathrm{r}}-10 W_{7}^{\mathrm{r}}-2 W_{8}^{\mathrm{r}}\right)(1-\cos \omega) \\
& \left.\quad+\left(\frac{\hat{m}}{m_{\mathrm{s}}-\hat{m}}\right)\left(3 L_{4}^{\mathrm{r}}+L_{5}^{\mathrm{r}}+4 L_{8}^{\mathrm{r}}+W_{5}^{\mathrm{r}}-3 W_{6}^{\mathrm{r}}-2 W_{8}^{\mathrm{r}}\right)(1-\cos \omega)\right] \\
& \quad-\frac{B_{0}}{64 \pi^{2} F_{0}^{2}}\left[\ln \left(\frac{\bar{m}_{K}^{2}}{\Lambda^{2}}\right)+1\right] \\
& -\frac{1}{192 \pi^{2} F_{0}^{2}\left(m_{\mathrm{s}}-\hat{m}\right)}\left[1-\frac{a W_{0}(1-\cos \omega)}{B_{0}\left(m_{\mathrm{s}}-\hat{m}\right)}\right] \\
& \left.\quad\left[\bar{m}_{\pi}^{2} \ln \left(\frac{\bar{m}_{\pi}^{2}}{\Lambda^{2}}\right)-3 \bar{m}_{\eta}^{2} \ln \left(\frac{\bar{m}_{\eta}^{2}}{\Lambda^{2}}\right)-\frac{1}{2}\left(3 \bar{m}_{\eta}^{2}-\bar{m}_{\pi}^{2}\right) \ln \left(\frac{\bar{m}_{K}^{2}}{\Lambda^{2}}\right)+\frac{3}{2}\left(\bar{m}_{\pi}^{2}+\bar{m}_{\eta}^{2}\right)\right]\right\} \\
& +\mathscr{O}\left(\Delta m^{3}, a^{2}\right) .
\end{aligned}
$$

We use the abbreviations $\Delta m \doteq m_{\mathrm{d}}-m_{\mathrm{u}}, \hat{m} \doteq\left(m_{\mathrm{u}}+m_{\mathrm{d}}\right) / 2$, and $F_{0}, B_{0}, W_{0}, L_{i}^{\mathrm{r}}$ and $W_{i}^{\mathrm{r}}$ are the low-energy constants familiar in Wilson chiral perturbation theory. The quantities

$$
\begin{aligned}
& \bar{m}_{\pi}^{2}=2 B_{0} \hat{m}+2 a W_{0} \cos (\omega)+\frac{a^{2} W_{0}^{2} \sin ^{2}(\omega)}{B_{0} \hat{m}}+\mathscr{O}\left(a^{3}\right) \\
& \bar{m}_{K}^{2}=B_{0}\left(\hat{m}+m_{\mathrm{s}}\right)+a W_{0}(1+\cos (\omega))+\frac{1}{2} \frac{a^{2} W_{0}^{2} \sin ^{2}(\omega)}{B_{0} \hat{m}}+\mathscr{O}\left(a^{3}\right) \\
& \bar{m}_{\eta}^{2}=\frac{1}{3}\left[2 B_{0}\left(\hat{m}+2 m_{\mathrm{s}}\right)+2 a W_{0}(2+\cos (\omega))+\frac{a^{2} W_{0}^{2} \sin ^{2}(\omega)}{B_{0} \hat{m}}\right]+\mathscr{O}\left(a^{3}\right)
\end{aligned}
$$

are the LO masses squared.

The pion mass splitting is induced by the $\pi^{0}-\eta$ mixing and is of order $\Delta m^{2}$. The presence of order $a$ terms even at full twist, $\cos \omega=0$, illustrates the necessity of a fourth quark flavour for automatic $\mathscr{O}(a)$ improvement.

Let us remark that in the pion masses themselves (which are not displayed in this conference contribution), terms proportional to the strange quark mass $m_{\mathrm{S}}$ show up. They are independent of $\omega$ and persist in the continuum limit. As the pions are made from up and down quarks, these terms are counterintuitive. However, the prefactors $L_{4}^{\mathrm{r}}$ and $L_{6}^{\mathrm{r}}$ have very small empirical values [23].

In case of the kaons the mass splitting between neutral and charged kaons in NLO to order $a$ 
turns out to be

$$
\begin{aligned}
& M_{K^{0}}^{2}-M_{K^{ \pm}}^{2}=B_{0} \Delta m\{1 \\
& +\frac{16 B_{0}}{F_{0}^{2}}\left[\left(-2 L_{4}^{\mathrm{r}}-L_{5}^{\mathrm{r}}+4 L_{6}^{\mathrm{r}}+2 L_{8}^{\mathrm{r}}\right) \hat{m}+\left(-L_{4}^{\mathrm{r}}-L_{5}^{\mathrm{r}}+2 L_{6}^{\mathrm{r}}+2 L_{8}^{\mathrm{r}}\right) m_{\mathrm{s}}\right] \\
& +\frac{8 a W_{0}}{F_{0}^{2}}\left[\left(-2 L_{5}^{\mathrm{r}}-6 W_{4}^{\mathrm{r}}-2 W_{5}^{\mathrm{r}}+6 W_{6}^{\mathrm{r}}+4 W_{8}^{\mathrm{r}}\right)\right. \\
& \left.\quad+\left(L_{5}^{\mathrm{r}}+4 W_{4}^{\mathrm{r}}+W_{5}^{\mathrm{r}}-4 W_{6}^{\mathrm{r}}-2 W_{8}^{\mathrm{r}}\right)(1-\cos \omega)\right] \\
& +\frac{1}{32 \pi^{2} F_{0}^{2}}\left[\frac{2 \bar{m}_{\eta}^{2}}{3} \ln \left(\frac{\bar{m}_{\eta}^{2}}{\Lambda^{2}}\right)\right. \\
& \left.\left.\quad+\frac{1}{B_{0}\left(m_{\mathrm{s}}-\hat{m}\right)} \frac{\bar{m}_{K}^{2}}{2}\left[\bar{m}_{\eta}^{2} \ln \left(\frac{\bar{m}_{\eta}^{2}}{\Lambda^{2}}\right)-\bar{m}_{\pi}^{2} \ln \left(\frac{\bar{m}_{\pi}^{2}}{\Lambda^{2}}\right)\right]\left(1-\frac{a W_{0}(1-\cos \omega)}{B_{0}\left(m_{\mathrm{s}}-\hat{m}\right)}\right)\right]\right\} \\
& +
\end{aligned}
$$

It has contributions linear in $\Delta m$ in contrast to the pions. These partly stem from pion and eta loops. Thus, for studies of the quark mass difference $\Delta m$, the kaon masses are suited much better than the pion masses.

To conclude, we have obtained the masses of the pseudoscalar mesons in Wilson chiral perturbation theory, taking into account the mass difference between up and down quarks. The mass splitting between neutral and charged pions, which is of order $\Delta m^{2}$, and the splitting between the kaon masses, which is of order $\Delta m$, are presented to order $a$ in the lattice spacing. Due to mixing with the $\eta$ meson, automatic $\mathscr{O}(a)$ improvement at maximal twist is lost.

\section{References}

[1] J. Gasser and H. Leutwyler, Quark Masses, Phys. Rept. 87 (1982) 77.

[2] G. Colangelo et al., Review of lattice results concerning low energy particle physics, Eur. Phys. J. C 71 (2011) 1695 [arXiv: 1011.4408 [hep-lat]].

[3] S. R. Sharpe, Applications of chiral perturbation theory to lattice QCD [arXiv: hep-lat/0607016].

[4] M. Golterman, Applications of chiral perturbation theory to lattice QCD [arXiv: 0912.4042 [hep-lat]].

[5] T. Blum, R. Zhou, T. Doi, M. Hayakawa, T. Izubuchi, S. Uno and N. Yamada, Electromagnetic mass splittings of the low lying hadrons and quark masses from $2+1$ flavour lattice QCD $+Q E D$, Phys. Rev. D 82 (2010) 094508 [arXiv: 1006.1311 [hep-lat]].

[6] S. Aoki et al., 1+1+1 flavour $Q C D+Q E D$ simulation at the physical point, Phys. Rev. D 86 (2012) 034507 [arXiv: 1205.2961 [hep-lat]].

[7] S. Borsanyi et al., Ab initio calculation of the neutron-proton mass difference, Science 347 (2015) 1452 [arXiv: 1406.4088 [hep-lat]].

[8] J. Gasser and H. Leutwyler, Chiral perturbation theory: Expansions in the mass of the strange quark, Nucl. Phys. B 250 (1985) 465. 
[9] R. Frezzotti, P. A. Grassi, S. Sint and P. Weisz, A local formulation of lattice QCD without unphysical fermion zero modes, Nucl. Phys. B (Proc. Suppl.) 83 (2000) 941 [arXiv: hep-lat/9909003].

[10] R. Frezzotti, P. A. Grassi, S. Sint and P. Weisz, Lattice QCD with a chirally twisted mass term, JHEP 0108 (2001) 058 [arXiv: hep-lat/0101001].

[11] R. Frezzotti and G. C. Rossi, Chirally improving Wilson fermions, I. O (a) improvement, JHEP 0408 (2004) 007 [arXiv: hep-lat/0306014].

[12] R. Frezzotti and G. C. Rossi, Twisted-mass lattice QCD with mass non-degenerate quarks, Nucl. Phys. B (Proc. Suppl.) 128 (2004) 193 [arXiv: hep-lat / 0311008 ].

[13] A. Shindler, Twisted mass lattice QCD, Phys. Rept. 461 (2008) 37 [arXiv: 0707.4093 [hep-lat]].

[14] R. Baron et al. [ETM Collaboration], Light hadrons from lattice QCD with light $(u, d)$, strange and charm dynamical quarks, JHEP 1006 (2010) 111 [arXiv: 1004.5284 [hep-lat]].

[15] K. Ottnad et al. [ETM Collaboration], $\eta$ and $\eta^{\prime}$ mesons from $N_{f}=2+1+1$ twisted mass lattice QCD, JHEP 1211 (2012) 048 [arXiv: 1206.6719 [hep-lat]].

[16] G. Münster and C. Schmidt, Chiral perturbation theory for lattice QCD with a twisted mass term, Europhys. Lett. 66 (2004) 652 [arXiv: hep-lat / 0311032 ].

[17] G. Münster, On the phase structure of twisted mass lattice QCD, JHEP 0409 (2004) 035 [arXiv: hep-lat/0407006].

[18] L. Scorzato, Pion mass splitting and phase structure in twisted mass QCD, Eur. Phys. J. C 37 (2004) 445 [arXiv: hep-lat/0407023].

[19] S. R. Sharpe and J. M. S. Wu, Twisted mass chiral perturbation theory at next-to-leading order, Phys. Rev. D 71 (2005) 074501 [arXiv: hep-lat/ 0411021 ].

[20] G. Münster and T. Sudmann, Twisted mass lattice QCD with non-degenerate quark masses, JHEP 0608 (2006) 085 [arXiv: hep-lat/0603019].

[21] G. Münster and T. Sudmann, Twisted mass chiral perturbation theory for $2+1+1$ quark flavours, JHEP 1104 (2011) 116 [arXiv: 1103.1494 [hep-lat]].

[22] S. Engelnkemper and G. Münster, Chiral perturbation theory for twisted-mass lattice QCD with three non-degenerate quark flavours [arXiv: 1507.02947 [hep-lat]].

[23] J. Bijnens and G. Ecker, Mesonic low-energy constants, Ann. Rev. Nucl. Part. Sci. 64 (2014) 149 [arXiv: 1405.6488 [hep-ph]]. 